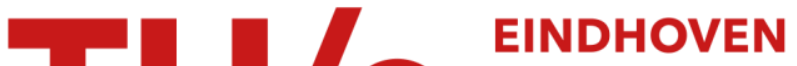 \\ UNIVERSITY OF \\ TECHNOLOGY
}

\section{Small signal analysis for analogue optical links with arbitrary optical transfer function}

Citation for published version (APA):

Rotman, R., Raz, O., \& Tur, M. (2004). Small signal analysis for analogue optical links with arbitrary optical transfer function. Electronics Letters, 4O(8), 504-505. https://doi.org/10.1049/el:20040355

DOI:

10.1049/el:20040355

Document status and date:

Published: 01/01/2004

Document Version:

Publisher's PDF, also known as Version of Record (includes final page, issue and volume numbers)

Please check the document version of this publication:

- A submitted manuscript is the version of the article upon submission and before peer-review. There can be important differences between the submitted version and the official published version of record. People interested in the research are advised to contact the author for the final version of the publication, or visit the $\mathrm{DOI}$ to the publisher's website.

- The final author version and the galley proof are versions of the publication after peer review.

- The final published version features the final layout of the paper including the volume, issue and page numbers.

Link to publication

\section{General rights}

Copyright and moral rights for the publications made accessible in the public portal are retained by the authors and/or other copyright owners and it is a condition of accessing publications that users recognise and abide by the legal requirements associated with these rights.

- Users may download and print one copy of any publication from the public portal for the purpose of private study or research.

- You may not further distribute the material or use it for any profit-making activity or commercial gain

- You may freely distribute the URL identifying the publication in the public portal.

If the publication is distributed under the terms of Article 25fa of the Dutch Copyright Act, indicated by the "Taverne" license above, please follow below link for the End User Agreement:

www.tue.nl/taverne

Take down policy

If you believe that this document breaches copyright please contact us at:

openaccess@tue.nl

providing details and we will investigate your claim. 


\section{Small signal analysis for analogue optical links with arbitrary optical transfer function}

\section{R. Rotman, O. Raz and M. Tur}

\begin{abstract}
A new analytical expression (the conversion matrix) for the effect of an arbitrary optical transfer function on small signal RF modulation is presented with applications to linear-frequency-modulated (LFM) signals. Chromatic dispersion of any order can be investigated, as well as wavelength-dependent insertion loss.
\end{abstract}

Introduction: The conversion matrix [1-3], connecting the optical intensity and phase variations at the output of an optical device with their corresponding input values, has been found useful in analysing laser and amplifier noise in analogue optical links and for computing the RF transfer function of various hybrid RF/optical systems. Previous works assumed the optical devices to be characterised by an all-phase transfer function [1-3], where the optical phase, was approximated by a finite order Taylor expansion around the optical centre frequency. However, present day optical devices have more complex transfer functions, including both phase and amplitude variations, at higher RF frequencies (millimetre waves) and with ever-wider RF bandwidths [4], requiring a more generalised matrix. This Letter derives such a matrix, valid for small signal modulation.

Theory: The field of an input optical signal, characterised by small intensity and phase variations can be approximately expressed by [1]

$$
E_{\text {in }}(t)=\sqrt{\langle I\rangle} \cdot\left[1+\Delta I_{\text {in }}(t) / 2\langle I\rangle+j \phi_{\text {in }}(t)\right] \cdot \exp \left[j \omega_{0} t\right]
$$

The propagation of this electromagnetic field through a linear optical medium will be investigated using the transfer function $H(\omega)=$ $E_{\text {out }}(\omega) / E_{\text {in }}(\omega)=A(\omega) \exp [-j \varphi(\omega)]$, where $E_{\text {in }}(\omega)$ and $E_{\text {out }}(\omega)$ are, respectively, the input and output complex amplitudes of the electric field at the optical frequency $\omega ; A(\omega)$ is the amplitude of $H_{o p t}(\omega)$, and $\varphi(\omega)$ is its phase. Assuming single tones for the intensity and phase variations,

$$
\begin{aligned}
\Delta I(t) /\left.2\langle I\rangle\right|_{\text {in }} & =m / 2 \cdot \cos \left(2 \pi f t+\phi_{R F}\right) \\
\phi_{\text {in }}(t) & =s / 2 \cdot \cos \left(2 \pi f t+\phi_{R F}\right)
\end{aligned}
$$

Using exponential representation for the cosines, the field at the output of the dispersive medium can be written as:

$$
\begin{aligned}
E_{\text {out }}(t) / \sqrt{\langle I\rangle}= & \left\{1+\frac{m}{4}\left[\exp \left[-\gamma_{+}(f)+j\left(2 \pi f t-\Delta \varphi^{+}(f)+\phi_{R F}\right)\right]\right]\right. \\
& +\frac{m}{4}\left[\exp \left[-\gamma_{-}(f)-j\left(2 \pi f t-\Delta \varphi^{-}(f)+\phi_{R F}\right)\right]\right] \\
& +j \frac{s}{4}\left[\exp \left[-\gamma_{+}(f)+j\left(2 \pi f t-\Delta \varphi^{+}(f)+\phi_{R F}\right)\right]\right] \\
& \left.+j \frac{s}{4}\left[\exp \left[-\gamma_{-}(f)-j\left(2 \pi f t-\Delta \varphi^{-}(f)+\phi_{R F}\right)\right]\right]\right\} \\
& \times A\left(\omega_{0}\right) \exp \left[j\left[\omega_{0} t-\varphi\left(\omega_{0}\right)\right]\right]
\end{aligned}
$$

where

$$
\begin{aligned}
\Delta \varphi^{+}(f) & =\varphi\left(\omega_{0}+2 \pi f\right)-\varphi\left(\omega_{0}\right) ; \\
\Delta \varphi^{-}(f) & =\varphi\left(\omega_{0}\right)-\varphi\left(\omega_{0}-2 \pi f\right) \\
\exp \left[-\gamma_{ \pm}(f)\right] & =A\left(\omega_{0} \pm 2 \pi f\right) / A\left(\omega_{0}\right)
\end{aligned}
$$

Using $\Gamma_{ \pm}(f)=\left[\exp \left(-\gamma_{+}(f)\right) \pm \exp \left(-\gamma_{-}(f)\right)\right] / 2$, (3) can be manipulated by dividing the output $E$ field into odd and even harmonic functions to obtain:

$$
\begin{aligned}
E_{\text {out }}(t) / \sqrt{\langle I\rangle}= & \left\{1+\frac{m}{2} \exp \left[-j \Phi_{\text {amp }}(f)\right]\right. \\
& \times\left\{\Gamma_{+}(f) \cos \left[2 \pi f t+\phi_{R F}+\Phi_{\text {phase }}(f)\right]\right. \\
& \left.+j \Gamma_{-}(f) \sin \left[2 \pi f t+\phi_{R F}+\Phi_{\text {phase }}(f)\right]\right\} \\
& +\frac{s}{2} \exp \left[-j \Phi_{\text {amp }}(f)\right] \cdot\left\{j \Gamma_{+}(f)\right. \\
& \times \cos \left[2 \pi f t+\phi_{R F}+\Phi_{\text {phase }}(f)\right] \\
& \left.\left.-\Gamma_{-}(f) \sin \left[2 \pi f t+\phi_{R F}+\Phi_{\text {phase }}(f)\right]\right\}\right\} \\
& \times A\left(\omega_{0}\right) \exp \left[j\left[\omega_{0} t-\varphi\left(\omega_{0}\right)\right]\right]
\end{aligned}
$$

where

$$
\begin{aligned}
\Phi_{\text {amp }}(f) & =0.5\left[\varphi\left(\omega_{0}+2 \pi f\right)+\varphi\left(\omega_{0}-2 \pi f\right)-2 \varphi\left(\omega_{0}\right)\right] \\
\Phi_{\text {phase }}(f) & =-0.5\left[\varphi\left(\omega_{0}+2 \pi f\right)-\varphi\left(\omega_{0}-2 \pi f\right)\right]
\end{aligned}
$$

Thus, the Fourier transforms of the output intensity and optical phase variations at the single RF frequency $f$, are related to their input values by:

$$
\begin{gathered}
{\left[\begin{array}{c}
{[\Delta I(f) / 2\langle I\rangle]} \\
\phi(f)
\end{array}\right]_{\text {out }}=\mathbf{T}(f)\left[\begin{array}{c}
{[\Delta I(f) / 2\langle I\rangle]} \\
\phi(f)
\end{array}\right]_{\text {in }}} \\
\mathbf{T}=\exp \left[j \Phi_{\text {phase }}(f)\right] \cdot\left[\begin{array}{c}
A_{1}(f) A_{2}(f) \\
-A_{2}(f) A_{1}(f)
\end{array}\right] \\
A_{1}(f)=\Gamma_{+}(f) \cos \left[\Phi_{a m p}(f)\right]-j \Gamma_{-}(f) \sin \left[\Phi_{a m p}(f)\right] \\
A_{2}(f)=\Gamma_{+}(f) \sin \left[\Phi_{a m p}(f)\right]+j \Gamma_{-}(f) \cos \left[\Phi_{a m p}(f)\right]
\end{gathered}
$$

The matrix formulation of (7) prescribes a method for the calculation of the effect of a general optical device on the transfer functions of both the RF complex amplitude and optical phase under small signal conditions, allowing for both $\mathrm{CW}$ and broadband inputs and making it possible to perform all calculations in the RF domain. Besides providing an analytical expression, this formulation should prove especially useful for concatenated optical devices, where the overall matrix is a product of the individual matrices. Equation (7) considerably simplifies when the amplitude of $H_{o p t}(\omega)$ is frequency independent $\left(\Gamma_{+}(f)=1 ; \Gamma_{-}(f)=0\right)$ :

$$
\mathbf{T}=\exp \left[j \Phi_{\text {phase }}(f)\right] \cdot\left[\begin{array}{cc}
\cos \left[\Phi_{a m p}(f)\right] & \sin \left[\Phi_{a m p}(f)\right] \\
-\sin \left[\Phi_{a m p}(f)\right] & \cos \left[\Phi_{a m p}(f)\right]
\end{array}\right]
$$

which for finite order dispersion, reduces to previously obtained expressions for the conversion matrix [1-3]. Equations (7) and (8) can be used to analytically study the effect of dispersion of any order on the performance of the RF link. When the initial excitation is pure intensity modulation, the even derivatives of $\varphi(\omega)$ (with respect to the optical frequency) affect the amplitude of the intensity through $\Phi_{\text {amp }}(f)$, while odd derivatives of the optical phase of the transfer function affect its phase through $\Phi_{\text {phase }}(f)$.

Application: Radar imaging systems often employ a chirped, linearfrequency-modulated (LFM) RF pulse of the form $\cos \left[\omega_{R F} t+\right.$ $\left.(B / 2 T) t^{2}\right]$ [5], where $\omega_{R F}$ is the pulse centre RF frequency, $B$ is the bandwidth, $T$ is the pulse duration, and the pulse exists between $-T / 2$ and $+T / 2$. After reception, the signal is matched-filtered to obtain the required range resolution (impulse response). For RF systems, the peak sidelobe level (PSL) of the weighted impulse response sets limitations on the acceptable phase and magnitude distortions of the RF transfer function (Fourier domain). When the phase or magnitude of the RF transfer function vary cosinusoidally with $\omega_{R F}$ with period $P$ obeying $P<B / 2$, the $P S L$ can be approximated by $P S L(\mathrm{~dB})=$ $20 \log _{10}(x / 2)$, where $x$ measures the amplitude of the phase or magnitude ripples [6]. Since the conversion matrix of a system/device transforms its optical characteristics into the RF domain, already known RF limitations can be now directly applied to photonic implementations of various RF subsystems, thereby defining the tolerances of the appropriate optical components.

Example: Assume an optical transfer function of the form:

$$
\begin{aligned}
H(\omega)= & \left(1+A \cos \left[\frac{2 \pi}{P_{\omega}}\left(\omega-\omega_{0}\right)+\theta_{A}\right]\right) \\
& \times \exp \left[j C \cos \left[\frac{2 \pi}{P_{\omega}}\left(\omega-\omega_{0}\right)+\theta_{C}\right]\right]
\end{aligned}
$$

where $A$ and $C$, respectively, measure the phase and magnitude ripples, $\omega_{0}$ is the optical frequency of the carrier, $P_{\omega}$ is the ripple period in optical frequency and $\theta_{A}$ and $\theta_{C}$ are offset angles. An optical signal, DSB modulated by an LFM waveform with an RF bandwidth $B \cdot T=10^{3}, B=4 P_{\omega}$, passed through the filter, followed by a weighted matched filter [7]. Assume $H(\omega)$ is phase only $(A=0)$. When the optical carrier is at the peak of the cosine $\left(\theta_{C}=0\right)$, we have from (6), (9): $\Phi_{\text {phase }}(f)=0$, and the optical phase ripple directly translates into an RF magnitude ripple, $1-\cos \left(\Phi_{a m p}(f)\right)$, the amplitude of which is of order $C^{2}$ (for $C \ll 1,(9)$ ), resulting in $P S L=20 \log _{10}\left(C^{2} / 2\right)(\mathrm{dB})$, or $P S L=-39 \mathrm{~dB}$ for $C=0.15$. To validate this value, we have calculated 
the effect of $H(\omega)$ on the PSL, numerically, by Fourier transforming the optically modulated signal $\left(E_{\mathrm{in}}(t),(1)\right.$ and $(2)$, with low modulation index), multiplying the transform by $H(\omega)$ and a weighted matched filter, and then back transforming to obtain the resulting impulse response. Results for various values of $A, C, \theta_{A}$ and $\theta_{C}$ appear in Fig. 1. In particular, for $A=\theta_{C}=0$, we get $-38.7 \mathrm{~dB}$, in very close agreement with our prediction (a similar numerical analysis, where all operations are performed in the RF domain by Fourier transforming the baseband LFM signal, multiplying the transform consecutively by $T_{11}(\omega)$ of (8), the matched filter and the weighting window and then back transforming, produced the same curves). Both the phase of $T_{11}$, as well as its magnitude, are important for accurate determination of the $P S L$. When the carrier is $\pi / 2$ from the peak, $\Phi_{a m p}(f)=0$, and the phase ripple directly translates to an RF phase ripple of amplitude $C$, resulting in $P S L=20 \log _{10}(C / 2)(\mathrm{dB})$, or $P S L=-22.5 \mathrm{~dB}$ for $C=0.15$, in excellent agreement with Fig. $1\left(A=0 ; \theta_{C}=\pi / 2\right)$.

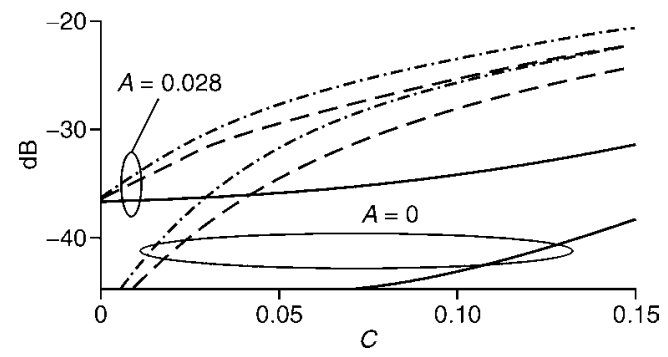

Fig. 1 PSL of impulse response of LFM signal, intensity modulating an optical carrier, after passing through optical filter of (9) for both $A=0$ and $A=0.028$ (0.25 $\mathrm{dB}$ in optical intensity)

$-\theta_{C}=0$

$---\theta_{C}=\pi / 4$

$\cdots \theta_{C}=\pi / 2$

Conclusion: A new conversion matrix, describing the effect of an arbitrary optical transfer function, having both amplitude and phase variations, on small signal $\mathrm{RF}$ modulation has been derived and discussed. The formulation allows calculations in the RF Fourier domain and can handle chromatic dispersion of arbitrary order. Using the conversion matrix, known tolerances of RF filters can be directly applied to optical filters, as demonstrated for the LFM case (DSM modulation). For modulation formats other than DSB, the conversion matrix also facilitates calculation using the whole upper half of the matrix. Its concatenation property should prove useful as well.

(C) IEE 2004

Electronics Letters online no: 20040355

30 January 2004 doi: 10.1049/el:20040355

R. Rotman, O. Raz and M. Tur (Faculty of Engineering, Tel-Aviv University, Tel Aviv 69978, Israel)

E-mail: rotman@eng.tau.ac.il

\section{References}

1 Wang. and Petermann, K.: 'Small signal analysis for dispersive optical fiber communication systems', J. Lightwave Technol., 1992, 10, (1), pp. 96-100

2 Cartaxao, A.V.T., and Morgado, J.A.P.: 'Relative intensity noise induced by fiber dispersion near zero-dispersion wavelength in linear single mode fibers', Proc. SPIE, 1998, 3491, pp. 521-526

3 Kaler, R.S, Kamal, T.S., and Sharma, A.K.: 'Approximate and exact small signal analysis for single-mode fiber near zero dispersion wavelength with higher order dispersion', Fiber and Integrated Optics, 2002, 21, (5), pp. 391-415

4 Rotman, R., Raz, O., and Tur, M.: 'Requirements for true time delay imaging systems with photonic components'. IEEE Phased Array Conf., Boston, MA, USA, 2003, pp. 193-198

5 Lacomme, P., Hardange, J.-P., Marchais, J.C., and Normant, E.: 'Air and spaceborne radar systems-an introduction' (William Andrew, Publisher, 2001), Chap. 16, pp. 266-271

6 Korman, N.I., Herman, E.B., and Ford, J.R.: 'Analysis of microwave antenna sidelobes', RCA Rev., 1952, 13, pp. 323-334

7 Jasik, H. (Ed.): 'Antenna Engineering Handbook' (McGraw Hill, 1961), Chap. 2, pp. 27 